\title{
A Dynamic Spectrum Access Approach in Energy-efficient Cognitive Wireless Networks
}

\author{
Weisong He \\ Department of Electronic information Engineering, Liangjiang International College, Chongqing \\ University of Technology \\ *heweisong@gmail.com
}

Keywords: Spectrum sharing, Dynamic spectrum access, Cognitive networking, Energy efficiency, Optimal model.

\begin{abstract}
Dynamic spectrum access can effectively improve network frequency resource utilization in current wireless networks. However, for energy-efficient cognitive wireless access networks, traditional dynamic spectrum access technologies face new challenges. This paper proposes a new dynamic spectrum access approach to improve both frequency resource utilization and network energy efficiency. We model this problem as an optimal model. In such a case, network frequency resource sharing and energy efficiency are combined into the optimal process to solve our model. Based on theory analysis, we propose our dynamic spectrum access algorithm to build highly energy-efficient cognitive wireless networks. Finally, we conduct a large amount of tests to validate our approach. Simulation results show that the proposed approach is effective and feasible.
\end{abstract}

\section{Introduction}

With the extensive applications and utilizations of wireless access technologies such as 3G, WiMax, and Wi-Fi, and so on, wireless frequency resources are becoming sparer and sparser [1-2]. At the same time, access equipment and network traffic are increasing rapidly. These further result in spectrum resources to become sparser. In turn, we will experience poor quality of experience and network performance [3-4]. Moreover, in such a case, network energy demands are also becoming larger and larger. The existing dynamic spectrum access technologies for cognitive wireless networking are difficult to effectively solve these problems [5-6]. Therefore, how to achieve frequency sharing and energy efficient dynamic spectrum access to cognitive wireless networks is an important problem to urgently be solved at present. This has become a significantly hot research topic in current communication communities [6-9].

Up to date, some approaches have been proposed to solve partly this problem. Spectrum sensing has been to achieve dynamic spectrum access [5], where authors assumed the idle status of the channel to follow a random distribution. Zhang et al. proposed the new method to maximize the spectrum utilization [10]. However, these methods only considered frequency sharing but did not considered network energy efficiency problem. Palmieri et al. [11] and Jiang et al. [3,7] proposed a new energy-efficient networking method to achieve the low energy consumption in wireless networks, which combined network energy efficiency, throughput, and interference to improve network performance. Xie et al. [12] studied the energy-efficient network resource allocation approach to realize the maximum frequency resource sharing in the mobile cellular network. These methods could attain the better frequency sharing between primary users and secondary users via minimizing the network energy consumption [13-16]. At the same time, they still hold larger energy overheads and lower frequency sharing. This motivates us to study a new dynamic frequency access method to solve the energy-efficient cognitive networking problem in wireless networks.

This paper studies the highly energy-efficient networking problem in dynamic spectrum accesses for cognitive wireless networks, which proposes a new dynamic spectrum access method to achieve the high frequency resource sharing and high network energy efficiency. Firstly, we discuss the dynamic frequency access problem in cognitive networks. The corresponding access model is 
proposed to characterize the utilization problem of the channel. Secondly, we model network energy consumption overheads in cognitive wireless networks. We study the collision avoidance and sleeping scheme to improve network energy consumption. By appropriate sleeping mechanism, we further reduce network energy consumption. Thirdly, we propose the energy efficiency model to maximize network through and minimize network energy consumption. The utility function is used to perform the appropriate collision avoidance and sleeping. The corresponding optimal model is proposed to build the dynamic spectrum access in energy-efficient cognitive wireless networking. We propose a dynamic frequency access algorithm to solve the model. Finally, Simulation results show that the proposed approach is feasible.

\section{Problem Statement}

This paper assume that our cognitive networking scenario includes a pair of primary users (PU) and $n$ pairs of secondary users (SU), where PUs have only two states: busy and idle. When PUs are busy, this denotes that the licensed channel is used. Generally, PUs' behaviors are independent in different time. Therefore, we use the exponential distribution to model the channel status as follows:

$$
p(t)=\alpha e^{-\alpha t} \quad 0<t
$$

According to Equation (1), we attain the bellow equation:

$$
F\left(t_{0}\right)=P\left[t \leq t_{0}\right]=1-e^{-\alpha t_{0}}, \quad t_{0} \geq 0
$$

For the distribution model of the channel utilization in (1)-(2), assume that variable $t$ denotes the time going away after PU changes from busy status to idle status. After the sensing of the SU lasts for the duration $t_{s}$, the following idle probability of the PU can be attained if the PU is idle at time $t$ :

$$
p^{s}(t)=\frac{1-\left(1-e^{-\alpha\left(t+t_{s}\right)}\right)}{1-\left(1-e^{-\alpha t}\right)}=e^{-\alpha t_{s}}
$$

After the sensing of the SU lasts for the duration $t_{t r}$, the idle probability of the PU is:

$$
p^{t r}(t)=\frac{1-\left(1-e^{-\alpha\left(t+t_{r r}\right)}\right)}{1-\left(1-e^{-\alpha t}\right)}=e^{-\alpha t_{t r}}
$$

where $s$ and $\operatorname{tr}$ express the sensing and transmitting behavior of the SU respectively, $t_{s}$ is the time length of SU sensing, $t_{t r}$ stands for the time length of the SU transmission.

Equations (3)-(4) model SUs' behaviors according to the channel status model in Equations (1)-(2). Accordingly, we use Equations (3) and (4) to perform the sensing and transmitting process of SUs. In this paper, our goal is to not only maximize the network frequency utilization, but also minimize network energy consumption. To save the energy, we use the sleeping mechanism to reduce energy overhead when PU and SUs do not transmit data packets. In such a case, The PU has two statuses: sleeping and transmitting, while the SUs include three statuses: sensing, sleeping, and transmitting.

Next, we discuss network energy overhead model for the PU and SUs. Without loss of generality, assume that the observed time duration is $t_{\text {len }}$. During the time length $t_{\text {len }}$, the energy overhead of the PU is denoted as:

$$
E_{p u, t_{l e n}}=E_{p u, t_{l e n}}^{s l}+E_{p u, t_{l e n}}^{t r}+E_{p u, t_{l e n}}^{c}+E_{p u, t_{l e n}}^{r e c}
$$

where $E_{p u, t_{\text {len }}}^{\mathrm{sl}}$ and $E_{p u, t_{\text {len }}}^{t r}$, respectively, denote PU's energy consumption when it stays in sleeping and transmitting statuses, $E_{p u, t_{l e n}}^{c}$ and $E_{p u, t_{l e n}}^{r e c}$ stands for basic circuit energy overhead and PU's receiving power. Given PU's sleeping power $P_{p u, s l}$ and sleeping time length $t_{p u, s l}, E_{p u, t_{l e n}}^{\mathrm{sl}}$ are denoted as:

$$
E_{p u, t_{l e n}}^{\mathrm{sl}}=P_{p u, s l} \times t_{p u, s l}=P_{p u, s l} \times \sum_{t \in\left[0, t_{l e n}\right]} l_{p u, s l}(t)
$$

Given PU's transmitting power $P_{p u, t}$ and transmitting time length $t_{p u, s l}, E_{p u, t_{l e n}}^{t r}$ are denoted as: 


$$
E_{p u, t_{l e n}}^{t r}=P_{p u, t r} \times t_{p u, t r}=P_{p u, t r} \times \sum_{t \in\left[0, t_{l e n}\right]} l_{p u, t r}(t)
$$

$E_{p u, t_{\text {len }}}^{\mathrm{c}}$ is expressed as follows:

$$
E_{p u, t_{l e n}}^{\mathrm{c}}=P_{p u, c} \times t_{l e n}
$$

$E_{p u, t_{\text {len }}}^{r e c}$ is expressed as follows:

$$
E_{p u, t_{\text {len }}}^{r e c}=P_{p u, \text { rec }} \times t_{p u, \text { rec }}=P_{p u, r e c} \times \sum_{t \in\left[0, t_{\text {len }}\right]} l_{p u, \text { rec }}(t)
$$

Similarly, SUs' energy consumption is denoted as follows:

$$
E_{s u, t_{l e n}}=E_{s u, t_{l e n}}^{\mathrm{s}}+E_{s u, t_{\text {len }}}^{t r}+E_{\text {su }, t_{\text {len }}}^{\mathrm{sl}}+E_{\text {su } \text { tlen }_{\text {len }}}^{c}+E_{\text {su, } t_{\text {len }}}^{r e c}
$$

where $E_{\text {su, } t_{\text {len }}}^{\mathrm{s}}, E_{\text {su, } t_{\text {len }}}^{\text {tr }}$, and $E_{\text {su, } t_{\text {len }}}^{s l}$, respectively, denote SUs' energy consumption when it stays in the sensing, transmitting, and sleeping statuses, $E_{s u, t_{l e n}}^{c}$ and $E_{s u, t_{\text {len }}}^{r e c}$ is SU's basic circuit energy overhead SUs' receiving energy consumption. For SU $i$, given the sensing, transmitting, sleeping, basic circuit, and receiving power $P_{s u, s}^{i}, P_{s u, t r}^{i}, P_{s u, s l}^{i}, P_{s u, c}^{i}$, and $P_{s u, r e c}^{i}$, and the corresponding time length $t_{s u, s}^{i}, t_{s u, t r}^{i}, t_{s u, s l}^{i}, t_{s u, c}^{i}$ and $t_{s u, r e c}^{i}, E_{s u, t t_{l e n}}^{\mathrm{s}}$ can be denoted as:

$$
E_{s u, t_{l e n}}^{\mathrm{s}}=\sum_{i}^{n}\left(P_{s u, s}^{i} \times t_{s u, s}^{i}\right)=\sum_{i}^{n}\left(P_{s u, s}^{i} \times \sum_{t \in\left[0, t_{l e n}\right]} l_{s u, s}^{i}(t)\right)
$$

$E_{\text {su, } t_{l e n}}^{t r}$ can be denoted as:

$$
E_{s u, t_{\text {len }}}^{t r}=\sum_{i}^{n}\left(P_{s u, t r}^{i} \times t_{s u, t r}^{i}\right)=\sum_{i}^{n}\left(P_{s u, t r}^{i} \times \sum_{t \in\left[0, t_{l e n}\right]} l_{s u, t r}^{i}(t)\right)
$$

$E_{\text {su, } t_{\text {len }}}^{s l}$ can be denoted as:

$$
E_{s u, t_{l e n}}^{\mathrm{sl}}=\sum_{i}^{n}\left(P_{s u, s l}^{i} \times t_{s u, s l}^{i}\right)=\sum_{i}^{n}\left(P_{s u, s l}^{i} \times \sum_{t \in\left[0, t_{l e n}\right]} l_{s u, s l}^{i}(t)\right)
$$

$E_{\text {su, } t_{l e n}}^{c}$ can be denoted as:

$$
E_{s u, t_{\text {len }}}^{\mathrm{c}}=\sum_{i}^{n}\left(P_{s u, c}^{i} \times t_{s u, c}^{i}\right)=\sum_{i}^{n}\left(P_{s u, c}^{i} \times \sum_{t \in\left[0, t_{l e n}\right]} l_{s u, c}^{i}(t)\right)
$$

$E_{s u, t_{l e n}}^{r e c}$ can be denoted as:

$$
E_{\text {su, } t_{\text {len }}}^{\mathrm{rec}}=\sum_{i}^{n}\left(P_{\text {su,rec }}^{i} \times t_{\text {su, rec }}^{i}\right)=\sum_{i}^{n}\left(P_{\text {su, rec }}^{i} \times \sum_{t \in\left[0, t_{\text {len }}\right]} l_{\text {su, rec }}^{i}(t)\right)
$$

Now, the channel status model has been discussed. PU's and SUs' energy consumption models have been proposed to characterize network energy overhead. In the following, we take the network energy efficiency as the objective function to build the dynamic spectrum access model for cognitive networks. Different form pervious methods, we consider the total energy overheads including PU's and SUs' energy consumption, and total delivered bit information. During the observed duration $t_{\text {len }}$, our model is described as: 


$$
\begin{cases}\max & \frac{\left.r_{p u, p a c} \times \sum_{t \in[0, t \text { ten }}\right]}{l_{p u, t r}(t)+\sum_{i=1}^{n}\left(r_{s u, p a c}^{i} \times \sum_{t \in\left[0, t_{l e n}\right]} l_{s u, t r}^{i}(t)\right)} \\ \text { s.t. } \quad & t_{l e n}=t_{p u, s l}+t_{p u, t r}+t_{p u, r e c}+E_{s u, t_{l e n}} \\ & t_{l e n}=t_{s u, s}+t_{s u, s l}+t_{s u, t r}+t_{s u, r e c} \\ & P_{p u, s l}, P_{p u, t r}, P_{p u, r e c} \leq P_{p u} \\ & P_{s u, s}^{i}, P_{s u, t r}^{i}, P_{s u, s l}^{i}, P_{s u, r e c}^{i} \leq P_{s u}^{i} \\ & \delta_{p u, S I N R} \geq \eta_{p u, S I N R}, \quad \delta_{s u, S I N R}^{i} \geq \eta_{s u, S I N R}^{i}\end{cases}
$$

where $r_{p u, p a c}$ and $r_{s u, p a c}^{i}$ denote PU's and SU's packet rate.

Equation (16) model the dynamic spectrum access problem in energy-efficiency cognitive networks. However, it is very difficult to directly solve this model. We propose a heuristic algorithm to attain the optimal solver to the model. The detailed steps of our algorithm are as follows:

Step 1: Give PU's maximum transmitting power $P_{p u}$, the maximum transmitting power $P_{s u}^{i}$ of SU $i$, PU's SINR threshold $\eta_{p u, S I N R}$, the SINR threshold $\eta_{s u, S I N R}^{i}$ of SU $i$, initialize sensing time $t_{s 0}$, transmitting threshold $\beta$.

Step 2: If SUs sense that the channel is idle, let $t=0$ and attain the idle probability $p^{s}(t)$ of the channel according to Equation (3).

Step 3: If $p^{s}(t)>\beta$, SUs begin to transmit data packets in the transmitting window and let PU begins to sleeping for the time length $l_{p u, s l}^{w}$. Or otherwise, go to Step 9.

Step 4: According to Equation (4), compute idle probability $p^{t r}(t)$. Calculate maximum transmitting time length $t_{\max }^{t r}$ in terms of the probability $p^{t r}(t)$.

Step 5: If PU has no data to transmit, let PU sleep for the time length $l_{p u, s l}^{w}$, and compute SUs start to transmit data packets in the transmitting window $t_{w, t r}$. Or otherwise PU sends data packets and go back to Step 3.

Step 6: If SUs receive acknowledge information from receivers, then let $t=t+t_{w, t r}$ and go back step 5. Or otherwise, recalculate the maximum transmitting window $h_{t r}^{t}$.

Step 7: Let SUs awake after sleep $h_{t r}^{t}$, and use avoidance mechanisms to send data packets, let $t=t+h_{t r}^{t}$.

Step 8: If $h_{t r}^{t} \leq t_{\max }^{t r}-t+1$, go back step 7 .

Step 9: Continue to sense in the next duration $t_{s 0}$. If PUs are busy, calculate SUs' sleeping window $l_{s u, s l}^{w}$ and let SUs sleep.

Step 10: SUs sense the channel. If PUs are busy, go back step 9. Or otherwise go back Step 2.

\section{Simulation Results and Analysis}

Now we perform a series of experiments to verify our approach for energy-efficient cognitive networking. We discuss the impact of parameters on network energy efficiency. At the same time, we also the impact of sensing time length, punishment factor, and collision factor on network energy efficiency performance.

Fig. 1 plots the impact of parameter $\alpha$ in equations (1)-(4) on network energy efficiency. It is very interesting that when $\alpha$ becomes larger, the energy efficiency of cognitive networks gradually increases. This is led by the following case: The larger $\alpha$ is the larger network throughput. In such a 
case, network energy efficiency gets higher. We also find that the bigger sending window holds the higher energy efficiency. This is because the larger sending window can be used to send more data packets. Fig. 2 plots the impact of the sending time length on network energy efficiency. We see that that when the sensing time length becomes larger, the energy efficiency of cognitive networks gradually drops. This is led by the following case: The longer sensing time length is, the lower network throughput is and the larger network energy consumption. Likely, Fig. 2 shows that the bigger sending window holds the higher energy efficiency. This show our method is effective.

Fig. 3 shows the impact of the punishment factor on network energy efficiency. It is obvious that when the punishment factor becomes large, the network energy efficiency decreases. This is because the larger punishment factor blocks SUs to transmit data packets. Accordingly, the network throughput gets low while the energy is still heavily consumed. Likely, Fig. 3 shows that the bigger
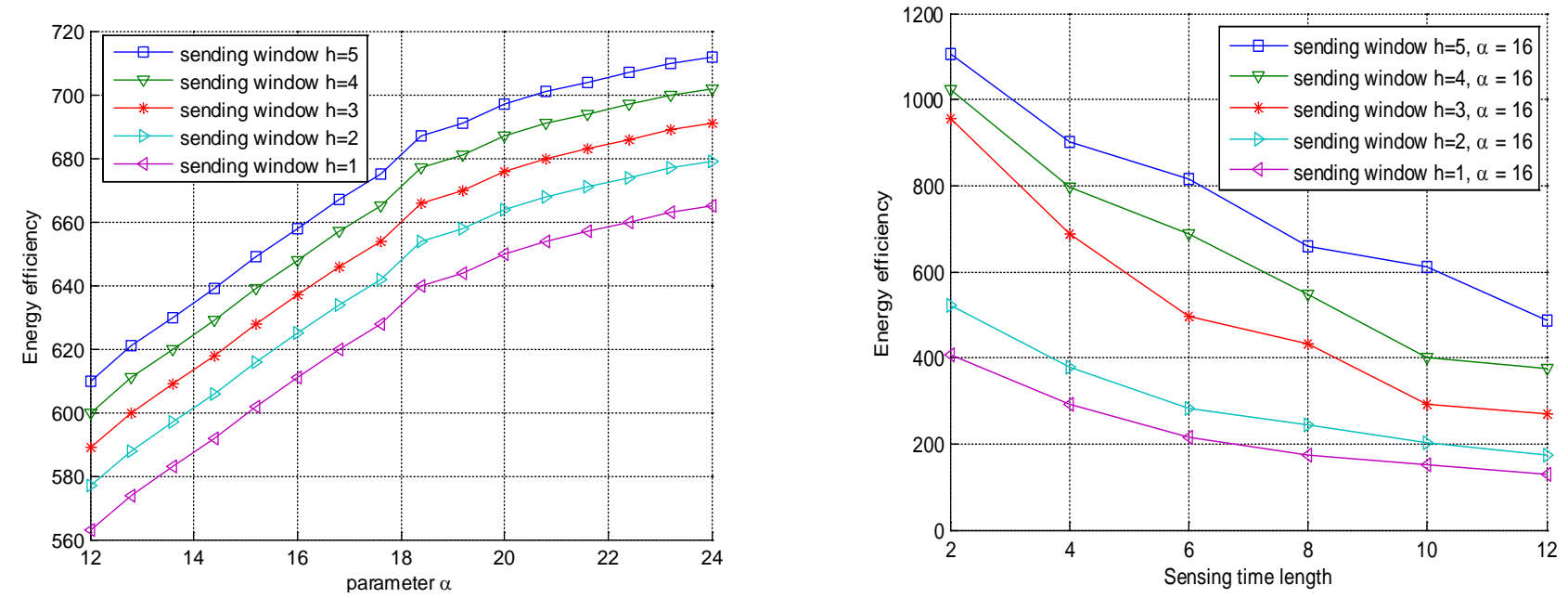

Fig. 1 The impact of parameters on energy efficiency. Fig. 2 The impact of sensing time length on energy efficiency.

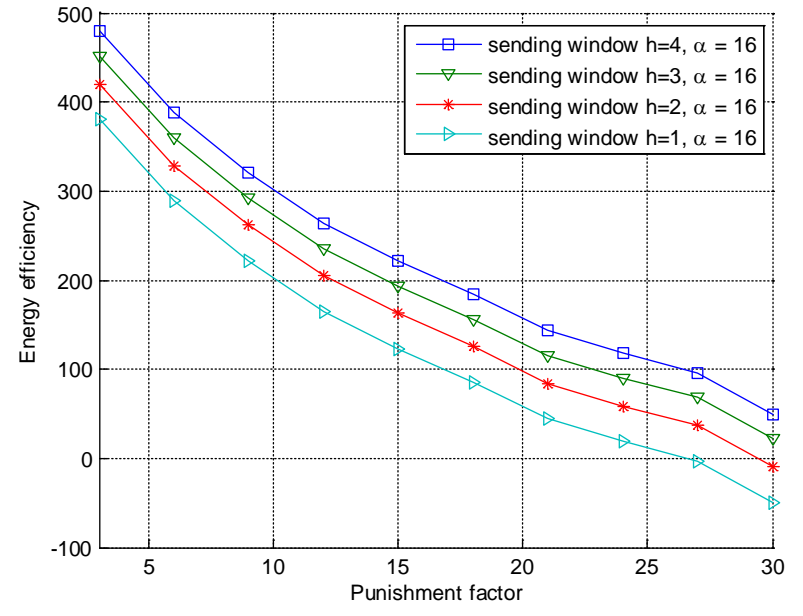

Fig. 3 The impact of punishment factor on energy efficiency.

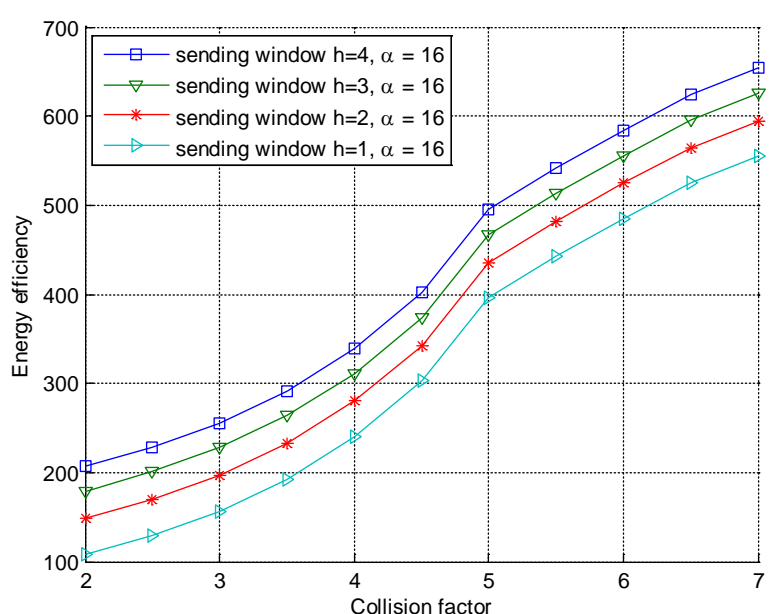

Fig. 4 The impact of collision factor on energy efficiency.

sending window also holds the higher energy efficiency. This indicates that our method holds the better performance for different punishment factor. Fig. 4 plots the impact of the collision factor on network energy efficiency. We find that when the collision factor grows, the network energy efficiency increases. This is because the larger collision factor let SUs more successfully transmit data. This make network throughput get large. Likely, Fig. 4 shows that the bigger sending window also holds the higher energy efficiency. This indicates that our method is feasible. Fig. 3 and 4 further indicate that our method can attain better energy efficiency performance. 


\section{Conclusions}

This paper proposes a new dynamic spectrum access approach for highly energy-efficient cognitive networking. We study the more practical dynamic spectrum access scenario with a pair of PUs and several pairs of SUs. The channel status model and network energy consumption models are proposed to characterize network energy overhead. The PU and SUs sleeping scheme and collision avoidance mechanism are introduced to improve energy efficiency. Simulation results show that the proposed approach is effective and feasible.

\section{Acknowledgement}

This paper is supported by the Scientific and Technological Research Program of Chongqing Municipal Education Commission (Grant No. KJ120822).

\section{References}

[1] Y. Lin, K. Liu, H. Hsieh. On using interference-aware spectrum sensing for dynamic spectrum access in cognitive radio networks. IEEE Transactions on Mobile Computing, 2012, online available.

[2] D. Jiang, Z. Xu, J. Liu, et al. An optimization-based robust routing algorithm to energy-efficient networks for cloud computing. Telecommunication Systems, 2016, 63(1): 89-98.

[3] D. Jiang, P. Zhang, Z. Lv, et al. Energy-efficient multi-constraint routing algorithm with load balancing for smart city applications. IEEE Internet of Things Journal, 2016, pp(99): 1-12.

[4] M. Dong, K. Ota, A. Liu, et al. Joint optimization of lifetime and transport delay under reliability constraint wireless sensor networks. IEEE Transactions on Parallel and Distributed Systems, 2016, 27(1): 225-236.

[5] S. Huang, X. Liu, Z. Ding. Optimal transmission strategies for dynamic spectrum access in cognitive radio networks. IEEE Transaction on Mobile Computing, vol. 8, no. 12, 2009, pp. 1636-1648

[6] J. Chen, Y. Gong, M. Fiorani, et al. Optical interconnects at the top of the rack for energy-efficient data centers. IEEE Communications Magazine, 2015, 53(8): 140-148.

[7] D. Jiang, Z. Xu, Z. Lv. A multicast delivery approach with minimum energy consumption for wireless multi-hop networks. Telecommunication Systems, 2016, 62(4), 771-782.

[8] D. Jiang, L. Nie, Z. Lv, et al. Spatio-temporal Kronecker compressive sensing for traffic matrix recovery. IEEE Access, 2016, 4: 3046-3053.

[9] E. Jung and X. Liu. Opportunistic spectrum access in multiple primary user environments under the packet collision constraint. IEEE Transactions on Networking, vol. 20, no. 2, 2012, pp. 501-514.

[10]X. Zhang, H. Su. CREAM-MAC: Cognitive radio-enabled multi-channel mac protocol over dynamic spectrum access networks. IEEE Journal on Selected Topics in Signal Processing, vol. 5, no. 1, 2011, pp. 110-123.

[11] F. Palmieri, U. Fiore, S. Ricciardi, et al.. GRASP-based resource re-optimization for effective big data access in federated clouds. Future Generation Computer Systems, 2016, 54(2): 168-179.

[12] R. Xie, F. Yu, H. Ji. Energy-efficient spectrum sharing and power allocation in cognitive radio femtocell networks. In Proc. of INFOCOM’12, 2012, pp. 1665-1673.

[13] D. Jiang, W. Li, H. Lv. An energy-efficient cooperative multicast routing in multi-hop wireless networks for smart medical applications. Neurocomputing, 2016, online available. 
[14]B. Addis, D. Ardagna, A. Capone, et al. Energy-aware joint management of networks and cloud infrastructures. Computer Networks, 2014, 70(1): 75-95.

[15] R. Jhawar, V. Piuri, M. Santambrogio. Fault tolerance management in cloud computing: a system-level perspective. IEEE Systems Journal, 2013, 32(1): 288-297.

[16] T. Bakera, B. Al-Dawsaria, H. Tawfikb, et al. GreeDi:An energy efficient routing algorithm for big data on cloud. Ad Hoc Networks, 2015, 35(2): 83-96. 